University of Windsor

Scholarship at UWindsor

2010

\title{
The Effectiveness of Wrist Guards for Reducing Wrist and Elbow Accelerations Resulting From Simulated Forward Falls
}

Timothy A. Burkhart

David M. Andrews

University of Windsor

Follow this and additional works at: https://scholar.uwindsor.ca/humankineticspub

Part of the Kinesiology Commons

\section{Recommended Citation}

Burkhart, Timothy A. and Andrews, David M.. (2010). The Effectiveness of Wrist Guards for Reducing Wrist and Elbow Accelerations Resulting From Simulated Forward Falls. Journal of Applied Biomechanics, 26 (3), 281-289.

https://scholar.uwindsor.ca/humankineticspub/4

This Article is brought to you for free and open access by the Faculty of Human Kinetics at Scholarship at UWindsor. It has been accepted for inclusion in Human Kinetics Publications by an authorized administrator of Scholarship at UWindsor. For more information, please contact scholarship@uwindsor.ca. 


\title{
The Effectiveness of Wrist Guards for Reducing Wrist and Elbow Accelerations Resulting From Simulated Forward Falls
}

\author{
Timothy A. Burkhart and David M. Andrews
}

\begin{abstract}
The effectiveness of wrist guards and modifying elbow posture for reducing impact-induced accelerations at the wrist and elbow, for the purpose of decreasing upper extremity injury risk during forward fall arrest, has not yet been documented in living people. A seated human pendulum was used to simulate the impact conditions consistent with landing on outstretched arms during a forward fall. Accelerometers measured the wrist and elbow response characteristics of 28 subjects following impacts with and without a wrist guard, and with elbows straight or slightly bent. Overall, the wrist guard was very effective, with significant reductions in peak accelerations at the elbow in the axial and off-axis directions, and in the off-axis direction at the wrist by almost $50 \%$. The effect of elbow posture as an intervention strategy was mixed; a change in magnitude and direction of the acceleration response was documented at the elbow, while there was little effect at the wrist. Unique evidence was presented in support of wrist guard use in activities like in-line skating where impacts to the hands are common. The elbow response clearly shows that more proximal anatomical structures also need to be monitored when assessing the effectiveness of injury prevention strategies.
\end{abstract}

Keywords: upper extremity, in-line skating, injury

Individuals tend to instinctively extend their arms in front of their bodies when they fall, to protect their heads and torsos from serious injury (Hsiao \& Robinovitch, 1998). As the hands come into contact with the ground, a shock wave is initiated that travels through the hand to more proximal anatomical structures of the upper extremity, including the wrist and elbow. The inherent instability and high velocities characteristic of in-line skating places participants at a high risk of upper extremity injuries when they fall. It is estimated that of the $20-60 \%$ of all in-line skating related injuries that occur to the forearm or wrist (distal radius) (Public Health Agency of Canada, 2000; Jerosch et al., 1998; Houshian \& Andersen, 2000), $50 \%$ will result in fracture, with $64 \%$ of all fractures resulting in orthopedic surgery (Houshian \& Andersen 2000; Houshian et al., 2001; Jaffe et al., 1997). While these injuries are generally not life threatening, they can lead to a number of serious post-fracture maladies, including median nerve compression, muscle ruptures, decreased grip strength, decreased range of motion, osteoarthritis, and extended periods of pain (Green \& Gay, 1956; Wong \& Pho, 1984; Stewart et al., 1985).

Wrist guards have been designed to attenuate the effects of the shock wave by absorbing and diverting

Timothy A. Burkhart (Corresponding Author) is with the Department of Kinesiology and the Department of Industrial and Manufacturing Engineering, University of Windsor, Windsor, ON, Canada. David M. Andrews is also with Department of Kinesiology and the Department of Industrial and Manufacturing Engineering, University of Windsor, Windsor, ON, Canada. the impact forces away from susceptible anatomical structures, through the implementation of a rigid (plastic) volar splint. While the force attenuating properties of wrist guards have been studied extensively, there is little agreement regarding their efficacy as an injury prevention mechanism. Schieber et al. (1996), Staebler et al. (1999) and Tan et al. (2001) all provide evidence that the risk of injury is decreased when in-line skaters are wearing wrist guards. In direct contrast is research by Cheng et al. (1995), Giacobetti et al. (1997), Greenwald et al. (1998) and Kim et al. (2006), who found that wrist guards were generally not capable of adequately absorbing impact forces and that serious injuries were still occurring even when wrist guards were worn.

Joint changes at the elbow have also been suggested as a method of damping the effects of impact forces. DeGoede \& Ashton-Miller (2002) found that by flexing the elbows during impact, an individual is capable of actively lowering the impact force peaks by approximately $60 \%$. These posture effects have led to the implementation of fall interventions (Lo et al., 2003), and have proven relatively successful at reducing ground reaction forces overall during upper extremity impacts.

Accelerometers have not been used extensively in the literature to measure the characteristics of the transmitted shock through the upper extremity following impact, despite considerable use for the lower extremity (e.g., Lafortune, Henning, \& Valiant, 1995) and the trunk (e.g., Wosk \& Voloshin, 1981). Radin et al. (1973) found a strong relationship between the peak magnitude of the transient shock wave and the risk of injury to subchondral bone at the knee joints of rabbits. Davis et al. (2004) and 
Milner et al. (2006) also found that females diagnosed with tibial stress fractures experienced greater tibial shock (measured as acceleration) and impact loading rates. The slope of the acceleration waveform provides a measure of the loading rate of the underlying structures. Increases in loading rate may result in a stiffened pathway, along which the shock wave will travel (Greenwald et al., 1998), and may result in an increased risk of fracture (Hansen et al., 2008; Milner et al., 2006). Different types of fracture may also occur depending on the loading rate (Porta, 2005).

To date, studies that have been conducted to establish the efficacy of wrist guards and elbow angle changes for attenuating potentially injurious impact forces have relied on epidemiological, cadaveric, or force plate data alone. The response of proximal anatomical structures of the upper extremity (i.e., the wrist and elbow) has not been studied in living people. Therefore, the purpose of the current study was to determine the effectiveness of two intervention strategies, a wrist guard and elbow joint posture changes, for attenuating the impact force effects on the upper extremities of living people, by analyzing the acceleration response directly at the wrist and elbow.

\section{Methods}

\section{Participants}

A novel seated human pendulum apparatus (see description below) was designed to simulate the flight phase of a forward fall, and deliver bilateral impacts to the upper extremities of 28 ( 15 male and 13 female) subjects (mean
[SD]): age, 23.1 (2.31) years; mass, $72.4(13.2) \mathrm{kg}$; and height, $1.7(0.1) \mathrm{m}$. Subjects were recruited from the University of Windsor kinesiology student population, and all methods and procedures were approved by the Research Ethics Board at the University of Windsor.

\section{Pendulum Apparatus}

The pendulum apparatus $(77 \mathrm{~cm} \times 53 \mathrm{~cm} \times 30 \mathrm{~cm})$ consisted of a perforated angle iron (2.5-cm-wide stock) frame, with a 6-mm plywood seat pan and back rigidly bolted to it, covered in 3-mm foam padding. The entire apparatus was suspended from the ceiling of the laboratory by four aircraft cables, such that the seat sat at rest approximately $0.6 \mathrm{~m}$ above the ground (Figure 1 ). The seat was designed so that individuals faced toward the impact surface and the angle between the trunk and the horizontal was maintained at approximately $110-120^{\circ}$; a trunk posture characteristic of forward falls (Chiu \& Robinovitch, 1998; DeGoede \& Ashton-Miller, 2002). Subjects were secured by two 5-cm nylon straps-one around the hips and the seat back, and one around the thighs and under the seat to support the legs comfortably in a flexed position.

\section{Impact Apparatus}

Two force plates (AMTI-OR6-6-1000, A-Tech Instruments Ltd, Scarborough ON, Canada, $1000 \mathrm{~Hz}$ natural frequency), were mounted to 2 -cm-thick steel plates $(46 \mathrm{~cm} \times 51 \mathrm{~cm}$, the bottoms located $83 \mathrm{~cm}$ above the floor) and were bolted rigidly side-by-side to a steel impact frame $(153 \mathrm{~cm} \times 122 \mathrm{~cm} \times 127 \mathrm{~cm})$, which in

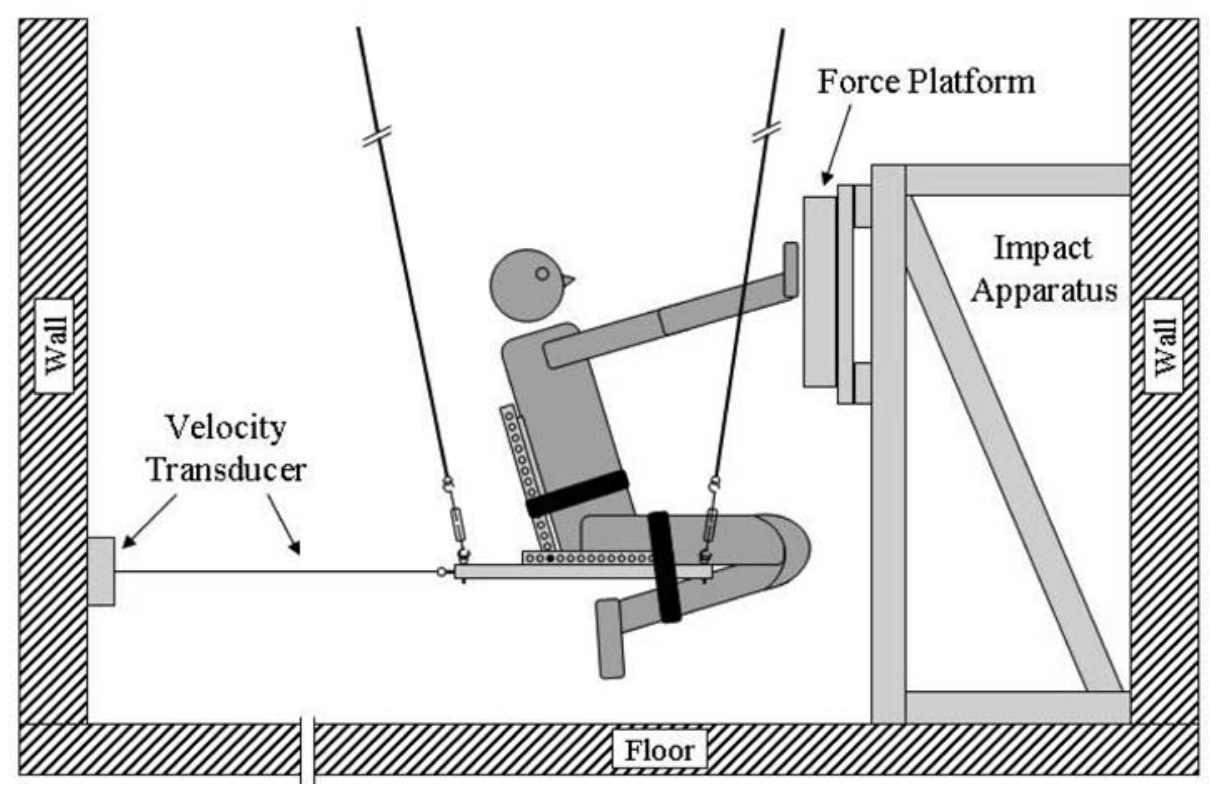

Figure 1 - An illustration of the experimental setup from a lateral view showing the seated human pendulum, the impact apparatus and the location of the velocity transducer and force platforms. The diagram reflects the positioning of the participant during an unguarded straight arm condition just before impact. Note that there were two force platforms, one for each hand to impact. 
turn was rigidly secured to the concrete floor and wall (Figure 1). Two pieces of steel tubing $(6.54 \mathrm{~cm} \times 6.54$ $\mathrm{cm})$ were placed between the force plates and the impact frame, thereby providing space for the knees below the force plates so they did not contact the impact apparatus before the hands struck the force plates during testing (see Procedures below).

\section{Instrumentation}

Impact reaction force (IRF) data (normal force plate forces) were used in conjunction with the pendulum velocity from a velocity transducer (Figure 1; Celesco DV301, Don Mills, ON, Canada), to ensure that impacts were occurring within previously documented ranges (Chiu \& Robinovitch, 1998). The angle of the right elbow joint was monitored using an electrogoniometer (Biometrics SG110 Biometrics Ltd., Gwent, UK), which was attached to the skin using double-sided tape (Figure 2).

Accelerations of the distal radius (representing the wrist) and the proximal ulna (representing the elbow) of the right arms of subjects were measured to determine the transient impact force effects with respect to the specified conditions. Two surface-mounted triaxial accelerometers (MMA1213D and MMA3201D, Freescale Semiconductor, Inc, Ottawa ON, Canada) with a range of $\pm 50 \mathrm{~g}$ and $\pm 40 \mathrm{~g}$, respectively, were used (Figure 2). The accelerometers were firmly attached to the skin using double sided tape and a normal preload of approximately 45 $\mathrm{N}$, which was applied using an elastic Velcro strap. The distal accelerometer was placed on the posterior surface of the distal forearm, medial to the radial styloid. The proximal accelerometer was placed over the olecranon process of the ulna. Accelerations were monitored along two axes at each location: parallel to the long axis of the forearm (axial direction), and at right angles to the axial direction (off-axis direction) (Figure 2). Accelerations along the third axis (medial-lateral direction) were negligible in magnitude due to the fact that the impacts were constrained to the sagittal plane. Consequently, accelerations in the medial-lateral direction will not be reported here and conclusions will be restricted to the axial and off-axis directions as described above. Three acceleration responses (dependent variables) were measured from the accelerometer waveforms (Figure 3): Peak Acceleration (PA), measured as the maximum acceleration magnitude; Acceleration Slope (AS), measured as the slope of the acceleration waveform taken between $30 \%$ and $70 \%$ of the peak acceleration (Holmes \& Andrews, 2006); and the Time to Peak Acceleration (TPA), measured as the time between impact and peak acceleration. Data collection was controlled by a custom LabView (National Instruments, Austin, TX, USA, version 7.1) software program. Acceleration data were filtered with an on-board switch capacitor fourth-order Bessel filter with a cut-off frequency of $1.5 \mathrm{~Hz}$. All data were sampled at $4096 \mathrm{~Hz}$ and $\mathrm{A} / \mathrm{D}$ converted with a 12 -bit card.

\section{Procedures}

Once subjects were seated and secured into the pendulum, they were asked to assume the position they would

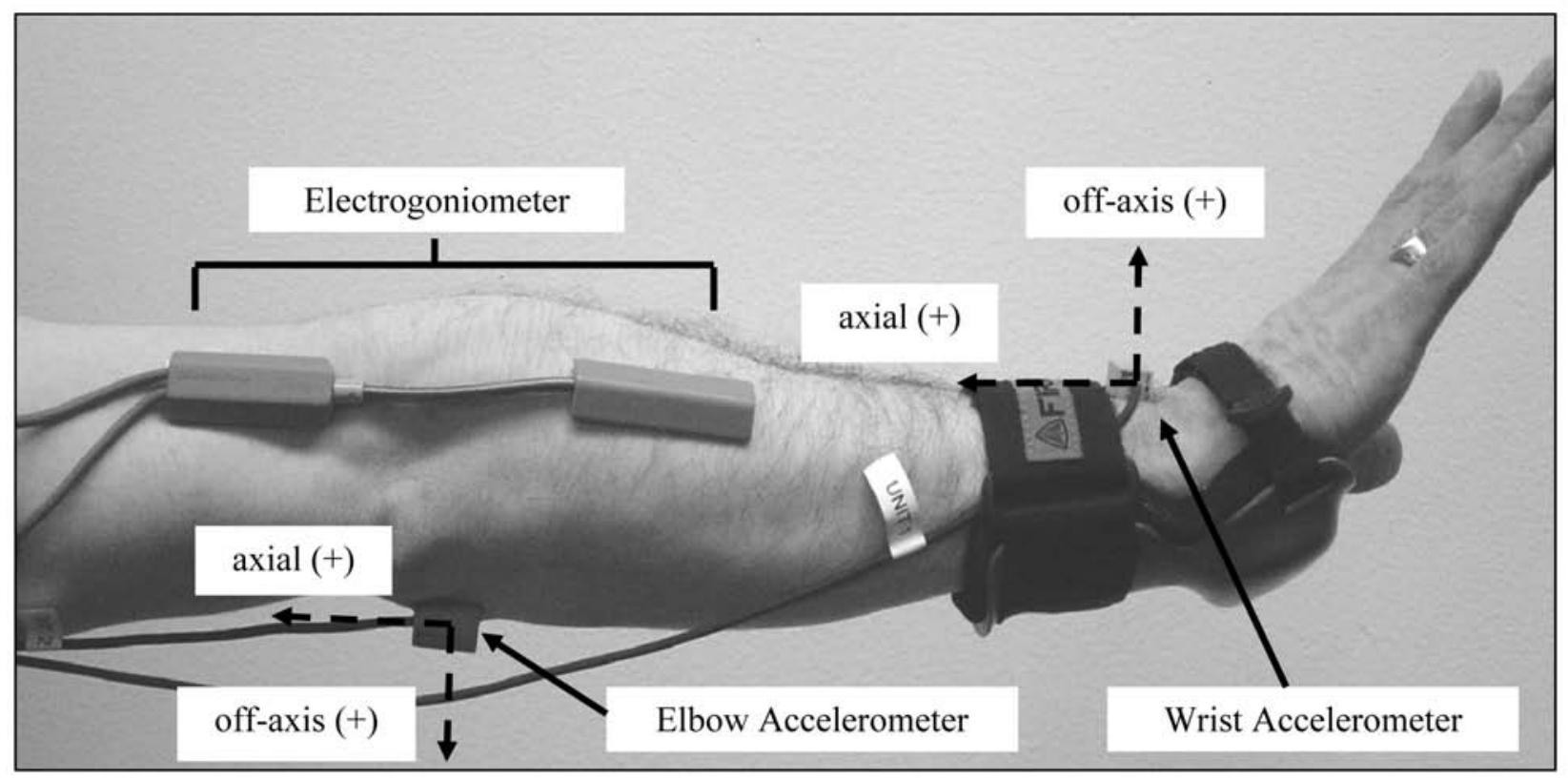

Figure 2 - An example of a subject's instrumented right forearm, showing the placement of the distal and proximal accelerometers and the electrogoniometer in the guarded, straight arm condition. The nylon straps used to preload the accelerometers are not shown so that the precise position of the accelerometers can be seen. 


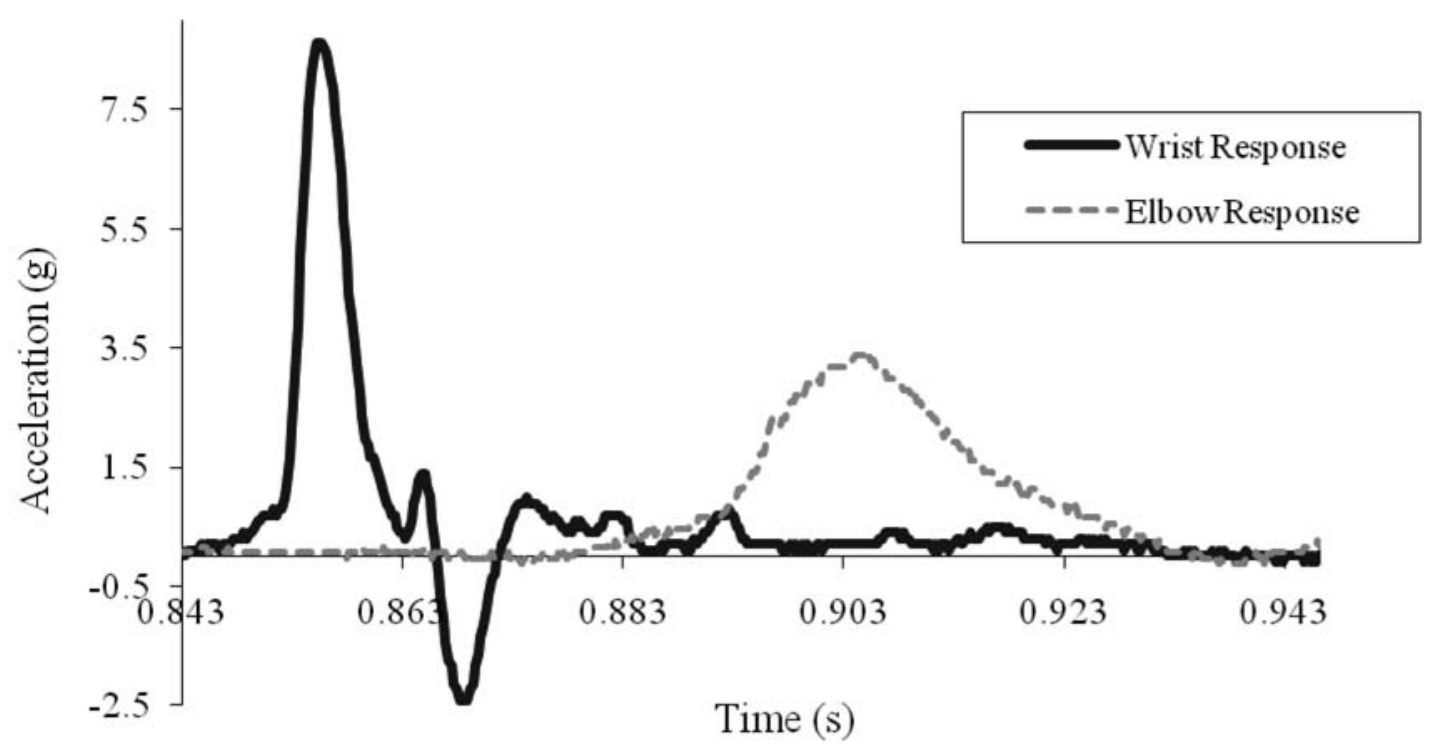

Figure 3 - Sample axial acceleration profiles recorded at the wrist and the elbow for an unguarded straight arm impact.

be in at impact with the force plates. This consisted of them placing their palms gently against the force plates with their elbows either completely extended (straight arm impact) or flexed at $168^{\circ}$ (natural arm impact), and their wrists extended between $30^{\circ}$ and $40^{\circ}$. Shoulder joint angle was not directly controlled for, but it was dictated by the position of the wrist and the elbow joint and was consistent within elbow angle and wrist guard conditions for each subject. In this position, the location of the base of the palm was marked on the force plates for both postures to provide a target for the subjects. The pull back distance for each subject was marked and held constant between trials such that the impact velocity was approximately $1 \mathrm{~m} / \mathrm{s}$ and the impact force was approximately $40-50 \%$ of the subjects' body weight (BW) (Chiu \& Robinovitch, 1998).

During testing, subjects sitting in the pendulum were released from their mark by an investigator when prompted by an auditory queue provided by the data collection program. While in the swing phase, between release and impact, subjects maintained the appropriate upper extremity postures. In the event that joint angles were not maintained throughout the impact, the condition was repeated. Feedback regarding elbow posture was provided to subjects during the swing phase via a computer monitor in their field of view. Three trials were recorded, for the straight arm and natural arm impacts, with and without a wrist guard in place (Firefly Sport Line wrist guard, model number: 065627). The wrist guard was secured to the hand with two dorsal straps such that the rigid plastic volar splint covered the palmar soft tissue and distal third of the forearm (Figure 2). All trials were randomized within wrist guard condition.

\section{Statistical Analyses}

A two-way ( 2 elbow angles $\times 2$ wrist guards) repeatedmeasures ANOVA was performed on the acceleration response variables (PA, AS, and TPA) in both the axial and off-axis directions at both the wrist and elbow joints to determine if differences existed between the various impact conditions. The means of the three trials for each elbow angle and wrist guard condition were used in the statistical analysis. To determine if subjects impacted their right and left hands similarly, independent sample $t$ tests were performed to compare the peak impact forces (Fmax) and the times of impact between the hands. The reliability of the pendulum method presented here was assessed with a repeated-measures ANOVA and intraclass correlation coefficients (ICCs) comparing the impact force recorded for each of the three trials. Good to excellent reliability was accepted at an ICC greater than 0.75 as per Portney \& Watkins (2000) and alpha was set at 0.05 for all statistical comparisons.

\section{Results}

Significant wrist guard effects were found at the wrist for $\mathrm{PA}_{\text {off }}, \mathrm{AS}_{\text {axial }}, \mathrm{AS}_{\text {off }}$ and TPA $\mathrm{Tff}_{\text {ond }}$ an the elbow for all acceleration response variables. Significant elbow angle effects were found at the wrist for $\mathrm{PA}_{\text {axial }}$, and $\mathrm{AS}_{\text {off }}$ and at the elbow for PA off $_{1}, \mathrm{AS}_{\text {off }}, \mathrm{TPA}_{\text {axial }}$ and TPA off (Table 1). Finally, no significant interaction effects were found between any of the variables.

While no significant differences between the unguarded and guarded conditions for peak axial acceleration and time to peak axial acceleration were found at the wrist, $\mathrm{AS}_{\text {axial }}$ increased significantly by a factor of more than 2 when the wrist guard was in place. In the off-axis direction, all acceleration responses showed a significant change on average when the wrist guard was in place: such that $\mathrm{PA}_{\text {off }}$ decreased by $49 \% ; \mathrm{AS}_{\text {off }}$ decreased by over $50 \%$; and TPA off increased by $15 \%$.

At the elbow, $\mathrm{PA}_{\text {axial }}$ was significantly decreased by approximately $2 g$ when the wrist guard was in place. 
Table 1 Mean (SD) acceleration responses at the elbow and wrist shown for each condition

\begin{tabular}{|c|c|c|c|c|c|c|c|c|}
\hline \multirow[b]{3}{*}{ Response } & \multicolumn{4}{|c|}{ Wrist } & \multicolumn{4}{|c|}{ Elbow } \\
\hline & \multicolumn{2}{|c|}{ Unguarded } & \multicolumn{2}{|c|}{ Guarded } & \multicolumn{2}{|c|}{ Unguarded } & \multicolumn{2}{|c|}{ Guarded } \\
\hline & Straight & Natural & Straight & Natural & Straight & Natural & Straight & Natural \\
\hline \multicolumn{9}{|l|}{ Axial } \\
\hline \multirow[t]{2}{*}{$\mathrm{PA}(g)$} & $11.1^{\mathrm{b}}$ & 12.1 & 12.0 & 12.1 & $5.9^{\mathrm{a}}$ & 5.6 & 3.8 & 3.8 \\
\hline & (1.6) & $(0.6)$ & $(1.1)$ & $(0.5)$ & $(0.3)$ & $(0.1)$ & $(0.3)$ & $(0.2)$ \\
\hline \multirow[t]{2}{*}{ AS $(g / s)$} & $2848.0^{\mathrm{a}}$ & 3546.1 & 7836.6 & 7632.8 & $817.6^{\mathrm{a}}$ & 941.2 & 331.6 & 349.6 \\
\hline & $(654.2)$ & (906.4) & $(566.3)$ & $(597.5)$ & (129.7) & (90.6) & (19.3) & (23.4) \\
\hline \multirow[t]{2}{*}{ TPA (ms) } & 18.7 & 16.9 & 17.4 & 17.6 & $21.4^{\mathrm{a}, \mathrm{b}}$ & 19.5 & 24.8 & 23.3 \\
\hline & $(0.7)$ & $(0.9)$ & (1.1) & $(1.0)$ & $(1.7)$ & $(2.0)$ & (1.5) & $(1.0)$ \\
\hline \multicolumn{9}{|l|}{ Off-Axis } \\
\hline \multirow[t]{2}{*}{ PA $(g)$} & $19.8^{\mathrm{a}}$ & 20.5 & 10.3 & 10.2 & $-1.2^{\mathrm{a}, \mathrm{b}}$ & 6.7 & -2.2 & 0.7 \\
\hline & $(4.0)$ & $(2.0)$ & $(0.5)$ & $(0.4)$ & $(1.0)$ & $(1.0)$ & $(0.6)$ & $(0.6)$ \\
\hline \multirow[t]{2}{*}{ AS $(g / s)$} & $5195.6^{\mathrm{a}, \mathrm{b}}$ & 5998.1 & 2590.7 & 2550.2 & $-600.2^{\mathrm{a}, \mathrm{b}}$ & 1853.9 & -242.2 & 311.60 \\
\hline & (390.4) & (510.1) & (161.9) & (155.3) & (282.2) & (381.7) & (156) & (139.1) \\
\hline \multirow[t]{2}{*}{ TPA (ms) } & $22.6^{\mathrm{a}}$ & 22.2 & 27.2 & 25.7 & $14.4^{\mathrm{a}, \mathrm{b}}$ & 12.2 & 20.8 & 16.1 \\
\hline & $(1.2)$ & (1.4) & $(0.8)$ & $(1.1)$ & $(0.6)$ & $(0.6)$ & (1.2) & $(0.6)$ \\
\hline
\end{tabular}

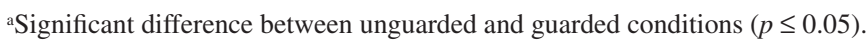

${ }^{\mathrm{b}}$ Significant difference between straight and natural elbow angle conditions $(p \leq 0.05)$.

No significant interaction effects were found.

Similarly, $\mathrm{AS}_{\text {axial }}$ was decreased by $60 \%$ and $\mathrm{TPA}_{\text {axial }}$ increased by $16 \%$. Wrist guards also had a significant effect on the off-axis acceleration parameters: peak accelerations decreased by about $3 g$ on average; mean acceleration slopes decreased by a factor of 20 ; and time to peak acceleration increased on average by $5 \mathrm{~ms}$.

At the wrist, elbow angle significantly affected the $\mathrm{PA}_{\text {axial }}$ response, such that mean peak acceleration increased between the straight arm $(11.5 \mathrm{~g})$ and the natural arm condition $(12.1 \mathrm{~g})$. Elbow angle did not have a significant effect on $\mathrm{PA}_{\text {off }}$ or on TPA $\mathrm{axial}_{\text {and }}$ and $\mathrm{TP}_{\text {off }} \mathrm{AS}_{\text {axial }}$ increased when the arm was impacted in a natural posture, but this change was not significant. However, $\mathrm{AS}_{\text {off }}$ increased significantly on average by approximately $10 \%$, from $3926.7 \mathrm{~g} / \mathrm{s}$ in the straight arm condition to 4323.3 $\mathrm{g} / \mathrm{s}$ when the subjects were impacted with natural arm postures.

$\mathrm{PA}_{\text {axial }}$ and $\mathrm{AS}_{\text {axial }}$ recorded at the elbow did not differ significantly between the straight arm and natural arm impacts. However, $\mathrm{PA}_{\text {off }}$ and $\mathrm{AS}_{\text {off }}$ were affected in both magnitude and direction by the two different elbow angles. Straight arm impacts resulted in a $\mathrm{PA}_{\text {off }}$ of $-1.8 \mathrm{~g}$, which is representative of a superiorly directed acceleration. However, natural arm impacts resulted in a significant increase in the magnitude of the mean $\mathrm{PA}_{\text {off }}$ to $3.7 \mathrm{~g}$, which occurred in the opposite direction than in the natural arm condition. A similar change was also recorded for $\mathrm{AS}_{\text {off }}$. Changes in elbow angle from a straight arm impact to a bent arm impact led to significant decreases in both $\mathrm{TPA}_{\text {axial }}(23 \mathrm{~ms}$ to $21 \mathrm{~ms})$ and $\mathrm{TPA}_{\text {off }}$ (18 $\mathrm{ms}$ to $14 \mathrm{~ms}$ ).
Subjects impacted the force plates at an average velocity of $1.01 \mathrm{~m} / \mathrm{s}$ and a force of $41 \%$ body weight (BW; $331 \mathrm{~N}$ ) across all conditions. The mean peak IRF for the right hand was significantly greater than for the left hand (Table 2). In $82 \%$ of the impacts, the right hand impacted approximately $0.4 \mathrm{~ms}$ before the left hand.

Mean peak IRFs were significantly lower when wrist guards were worn, and when the elbow was flexed in the natural arm position (Table 2). Furthermore, no significant differences were found between the three trials on any of the independent variables (impact force, PA, AS, TPA), and all ICCs were greater than 0.75 , suggesting excellent reliability between impact trials (Table 3 ).

\section{Discussion}

To the authors' knowledge, this is the first study to quantify the acceleration response of living people at the wrist and elbow following simulated forward falls. The response of the upper extremity to impact reaction forces was found to be dependent on the joint location (wrist vs. elbow), as well as the loading direction (axial vs. offaxis). Overall, the wrist guard studied was found to be very effective at attenuating impact induced accelerations at the elbow in both the axial and off-axis directions, and in the off-axis direction at the wrist.

The impact parameters in this study (velocity and force) were comparable in magnitude to previous work (Chiu \& Robinovitch, 1998; DeGoede \& Ashton-Miller, 2002). Maintaining these levels was important to provide some validation for the new pendulum methodology 
Table 2 Mean (SD) impact reaction forces and velocities by condition

\begin{tabular}{|c|c|c|c|c|c|c|c|c|c|}
\hline & \multirow[b]{2}{*}{ Overall } & \multicolumn{2}{|c|}{ Sex ${ }^{a}$} & \multicolumn{2}{|c|}{ Wrist Guard } & \multicolumn{2}{|c|}{ Elbow Angle $^{a}$} & \multicolumn{2}{|c|}{ Hand } \\
\hline & & Male & Female & Unguarded & Guarded & Straight & Natural & Left & Right \\
\hline \multirow[t]{2}{*}{ Force $(\% \mathrm{BW})$} & 41 & $45^{*}$ & 37 & $49 *$ & 32 & $42 *$ & 39 & $32 *$ & 41 \\
\hline & (2) & (3) & (3) & (2) & (1) & (2) & (2) & (7) & (10) \\
\hline \multirow[t]{2}{*}{ Velocity $(\mathrm{m} / \mathrm{s})$} & 1.01 & 1.00 & 1.02 & 1.01 & 1.01 & - & - & - & - \\
\hline & $(0.4)$ & $(0.04)$ & $(0.02)$ & $(0.03)$ & $(0.04)$ & & & & \\
\hline
\end{tabular}

${ }^{a}$ Force values are for the right extremity only because the left was not instrumented.

$* p \leq 0.05$.

Table 3 Mean (SD) impact reaction forces and acceleration response variables measured across the three trials. ICC values are included for between trials. No Significant differences were found for any variable. All data are from the instrumented right arm.

\begin{tabular}{lcccc}
\hline Variable & Trial One & Trial Two & Trial Three & ICC \\
\hline IRF $(\mathrm{N})$ & 328.6 & 332.5 & 335.0 & 0.99 \\
& $(98.9)$ & $(102.4)$ & $(99.0)$ & 0.99 \\
\hline PA $(g)$ & 11.8 & 11.8 & 11.9 & \\
& $(2.5)$ & $(2.5)$ & $(2.5)$ & 0.98 \\
\hline AS $(g / \mathrm{s})$ & 5423.1 & 5427.4 & 5495.0 & \\
& $(1664.6)$ & $(1739.3)$ & $(1741.0)$ & 0.79 \\
\hline TPA $(\mathrm{ms})$ & 17.8 & 17.0 & 18.1 & \\
& $(0.6)$ & $(0.6)$ & $(0.5)$ & \\
\hline
\end{tabular}

described in this study, and to ensure that subjects were impacting at levels that have previously been shown to be safe physiologically. Furthermore, the absence of between trial differences and the high ICCs speaks to the repeatability of the method used here, suggesting that the seated human pendulum is a reliable mechanism for simulating impacts to the upper extremity. Finally, while the effect that wrist guard use and elbow angles had on IRFs was comparable to that which has been reported elsewhere (Greenwald et al., 1998; DeGoede et al., 2002; Lo et al., 2003; Hwang et al., 2006) they tended to contradict those reported by Giacobetti et al. (1997). Several limitations exist however, in comparing the IRF results across previous wrist guard studies. For instance, no two studies have used the same wrist guards, and consequently, they have tested wrist guards that ranged in size, shape and materials. Secondly, of the studies that have used cadaver specimens, only a small portion of the distal forearm has been used with differing postures across studies. Finally, there is an absence of consistency across studies with respect to the postures attained by subjects at impact. These examples serve to illustrate why it is necessary to study the effects of shock waves on the upper extremity at anatomical locations away from the initial site of impact.

Peak IRFs measured at the hand/force plate interface varied by approximately $10 \%(134 \mathrm{~N})$ between the left and right hands. Although the majority of the subjects tested $(n=22)$ reported being right hand dominant, there was no significant difference between the left and right hand dominant subjects for the hand that produced the greatest force. Handedness also did not dictate which hand impacted first. Subjects were instructed to impact the force plates with the left and right hand simultaneously and were visually monitored to ensure that there was no obvious or intentional bias for one hand over the other. However, subjects may have been more aware of the right hand given that only the right upper extremity was instrumented, and may have subconsciously led with the right hand more often.

The force attenuating capacity of the wrist guard, at the wrist, was found to be dependent on the loading axis (axial vs. off-axis). Peak accelerations directed along the long axis of the forearm were generally unaffected by the use of a wrist guard. Kim et al. (2006) found similar results in the axial direction when they compared a bare hand to several different guarded conditions, including guards made from traditional plastic, Sorbothane, air cells and air bladders. They reported that on average, 2.5\% more force was transmitted when one of the protective materials was being used instead of a bare hand.

In comparison, there was a 50\% decrease in the peak acceleration in the off-axis direction after the wrist guard was implemented in the current study. In addition, $\mathrm{AS}_{\text {axial }}$ increased by $59 \%$ when the wrist guard was in use, while it was reduced by almost $50 \%$ in the off-axis direction. Taken together, these results suggest that instead of being 
directed through the hand (off-axis direction), the impact force was diverted down the volar splint, parallel with the long axis of the forearm and through the wrist. Greenwald et al. (1998) found similar results, reporting decreased impact forces between unguarded and guarded specimens, but little difference in the number of specimens that experienced a fracture or in the pattern of fractures when they did occur.

Model results from Troy \& Grabiner (2007) suggest that the loading axis has a significant influence on the risk and location of wrist fractures. They reported that it requires a smaller off-axis force to initiate an injury than when a force is acting axially. Although the current study only involved submaximal loading, the results suggest that wrist guards may be a suitable mechanism for protection against the relatively harmful off-axis forces, since comparable trends to Troy \& Grabiner (2007) were noted. Wrist guards may also provide a protective benefit because they tend to limit the degree to which the wrist is extended when impact occurs. An extended wrist at impact has been identified as a risk factor for wrist injuries (Hwang et al., 2006; Troy \& Grabiner, 2007).

The wrist guard was found to contribute to significant effects for all dependent variables measured at the elbow. The substantial changes in the acceleration responses at the elbow confirm the notion that wrist guards are capable of absorbing or redirecting impact reactions and accelerations before reaching the elbow. Past studies (Cheng et al.,1995; Schieber et al., 1996; Giacobetti et al., 1997, 1998; Staebler et al., 1999; Tan et al., 2001; Kim et al., 2006) have inferred injury risk at more proximal locations through analysis of hand force data only. However, this study is the first to quantify these responses at specific proximal locations away from the impact sites in living people, to assess the potential of injury reducing interventions that claim to attenuate the impact effect along the forearm.

The "natural angle" of $168^{\circ}$ (i.e., $12^{\circ}$ of flexion) that was used in this study has been shown to occur naturally when people impact the ground and try to protect themselves with outstretched arms (Hsiao \& Robinovitch, 1998; DeGoede \& Ashton-Miller, 2002; Lo et al., 2003). Changing the elbow angle is considered to be an active force attenuation mechanism because IRFs have been shown to decrease as a result (DeGoede et al., 2002; Lo et al., 2003). However, the response of the wrist in this study contradicts previously reported results. The natural elbow angle of $168^{\circ}$ may not have been a large enough change from the straight arm condition to be effective in reducing the impact effects at the wrist. In addition, in an attempt to control elbow angle across all subjects, impacts occurred to a statically flexed arm. This negates much of the active force absorption effect that is experienced when the elbow is allowed to flex freely at impact. Furthermore, keeping the wrist angle constant between the straight arm and natural arm conditions required the hand to impact lower on the force plate during natural arm impacts. Subsequently, shoulder flexion was decreased by approximately $5^{\circ}$ to achieve the desired wrist and elbow postures; decreasing the ability of the shoulder to actively react to, and absorb the IRF. In lower extremity impacts, the hip plays a major role in the attenuation of impact force, mainly by hip flexion (Zhang, Bates, \& Dufek, 2000). The shoulder may be responsible for similar force attenuation when the upper extremity is impacted.

With regards to the elbow, the off-axis response differed considerably from the axial response in that there was both a change in magnitude and direction for $\mathrm{PA}_{\text {off }}$ and $\mathrm{AS}_{\text {off }}$. Negative accelerations were recorded during a straight arm impact for both $\mathrm{PA}_{\text {off }}$ and $\mathrm{AS}_{\text {off }}$ On the other hand, when subjects were impacted with a slightly bent arm (natural condition), the acceleration response occurred in the positive direction and increased significantly in magnitude from the straight arm condition, up to $3.71 \mathrm{~g}$ and $1083 \mathrm{~g} / \mathrm{s}$ for $\mathrm{PA}_{\text {off }}$ and $\mathrm{AS}_{\text {off }}$, respectively. The change in loading direction may be a result of the localized impact of the distal humerus within the trochlear notch. When a straight arm impact occurs, the distal humerus is aligned with the long axis of the radius and ulna. Following the initial impact, and the positive peak acceleration, the negative axial acceleration may have been a result of the humerus impacting the ulna along the long axis. However, when the arm was flexed, the trochlea of the humerus would have rotated in the sagittal plane within the trochlear notch, and would have become more aligned with the off-axis of the elbow.

The off-axis (positive) loading of the elbow in the bent arm condition would occur as the trochlea of the humerus is impacted within the trochlear notch. This would result when the hand and forearm come to rest while the remainder of the body's mass is arrested following impact. This response helps to explain the many different types of elbow fractures reported in the literature (Ring et al., 1997; Houshian et al., 2001; Wake et al., 2004; Doornberg \& Ring, 2006). Wake et al. (2004) reported different fracture sites in the elbow based on elbow angles under static compressive loading (Figure 4). The elbow angle results reported here for dynamic loading, compliment the findings of Wake et al. (2004); as the magnitude of elbow flexion changed from $168^{\circ}$ to $180^{\circ}$, different anatomical structures are impacted.

The impact apparatus that was used in this study required subjects to remain in a seated position while they traveled toward the impact surface, similar to DeGoede et al. (2002). Although this orientation is not entirely representative of the positioning which occurs naturally during forward falls, the pendulum allowed for very consistent impact conditions to be presented. The seated human pendulum was also designed to meet the basic postural demands of a falling human, in terms of the trunk, shoulder and elbow angles at impact, as documented previously by Hsiao \& Robinovitch (1998) and Lo et al. (2003). It was observed in this study that the shoulder joint angle decreased between the natural and bent arm conditions, and although minimal (approx. $5^{\circ}$ on average), it should be recognized that active shoulder joint changes may have an effect on the response of the upper extremity to impact. However the joint changes observed here were consistent between subjects and 


\section{Trochlea of humerus}

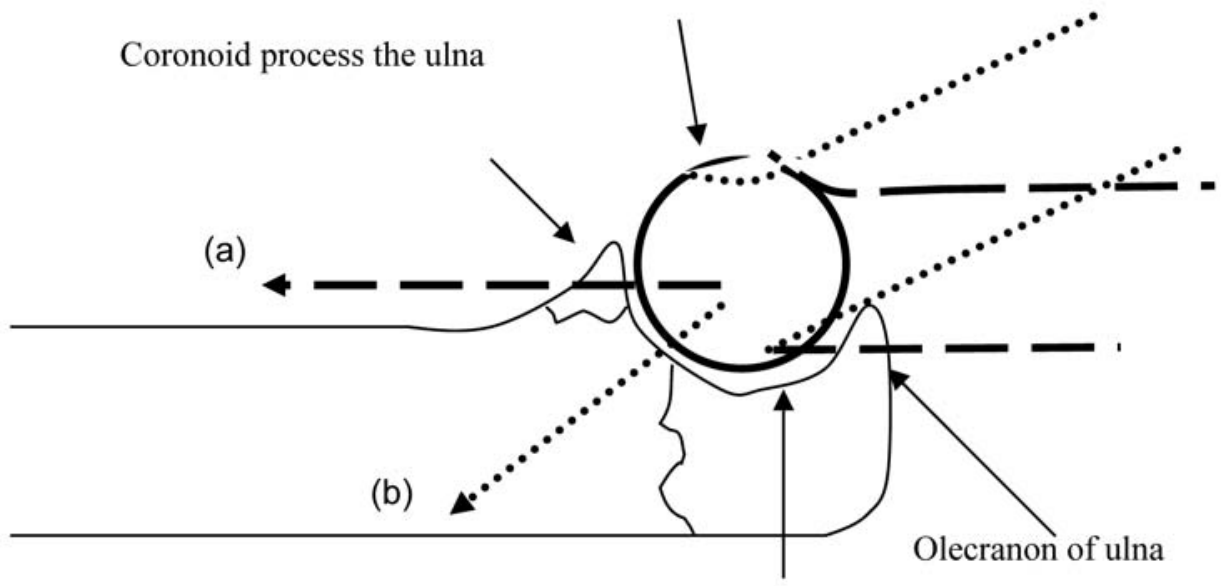

Trochlear notch

Figure 4-Schematic representation of the effects of different elbow angles on the loading axis at the elbow. Fracture of coronoid process results from a straight arm impact (dashed line [a]), and fractures to proximal structures, such as the olecranon, occur from bent arm impacts (dotted line [b]; Modified from Wake et al., 2004).

with upper extremity postures in similar work discussed elsewhere (Hsiao \& Robinovitch, 1998).

Another notable limitation of this study was the placement and type of accelerometers used to measure acceleration responses. Kim et al. (1993) and Lafortune et al. (1995) have shown that transient force waves are more accurately measured directly at the bone via bonemounted accelerometers. However, given the invasive nature of this technique, skin mounted accelerometers were used in the collection of acceleration responses at the wrist and elbow. Although skin-mounted accelerometers are more prone to picking up movement of the underlying soft tissue, precautions were taken in this study by preloading the transducers with the straps to ensure that they were firmly affixed as close to the bone as possible, thereby minimizing skin motion interference (Kim et al., 1993). The accelerometers were also placed over bony landmarks that did not interfere with the normal operation of the wrist guard (Figure 2).

The current study simulated a forward fall impact to the upper extremities so that the force attenuating characteristics of a wrist guard and elbow posture at impact could be assessed. Evidence was provided that suggests wrist guards may be beneficial in reducing the impact response at the elbow and in the off-axis direction at the wrist. These results also provide support for the notion that analyses of more proximal structures in the upper extremities may provide very useful information regarding fall injury prevention strategies.

\section{Acknowledgments}

This project was funded by NSERC. We also wish to thank Leah Okkema-Konwinski and Nikki Nolte for their assistance in data collection.

\section{References}

Cheng, S.L., Rajarantnam, K., Raskin, K.B., Hu, R.W., \& Axelrod, T.S. (1995). "Splint-top" fracture of the forearm: a descriptive of in-line skating injury associated with the use of protective wrist splints. The Journal of Trauma Injury Infection and Critical Care, 39, 1194-1197.

Chiu, J., \& Robinovitch, S.N. (1998). Prediction of upper extremity impact forces during falls on the outstretched hand. Journal of Biomechanics, 31, 1169-1176.

Davis, I., Milner, C.E., \& Hamill, J. (2004). Does increased loading during running lead to tibial stress fractures? A prospective study. Medicine and Science in Sports and Exercise, 36(Suppl. 5), S58.

DeGoede, K.M., Ashton-Miller, J.A., Schultz, A.B., \& Alexander, N.B. (2002). Biomechanical factors affecting the peak hand reaction force during the bimanual arrest of a moving mass. Journal of Biomechanical Engineering, 124, 107-112.

DeGoede, K.M., \& Ashton-Miller, J.A. (2002). Fall arrest strategy affects peak hand impact force in a forward fall. Journal of Biomechanics, 35, 843-848.

Doornberg, J.N., \& Ring, D. (2006). Coronoid fracture patterns. The Journal of Hand Surgery, 31, 45-52.

Giacobetti, F.B., Sharkey, P.F., Bos-Giacobetti, A., Hume, E.L., \& Taras, J.S. (1997). Biomechanical analysis of the effectiveness of in-line skating wrist guards for preventing wrist fractures. American Journal of Sports Medicine, 25, 223-225.

Greenwald, R.M., Janes, P.C., Swanson, S.C., \& McDonald, T.R. (1998). Dynamic impact response of human cadaveric forearms using a wrist brace. American Journal of Sports Medicine, 26, 825-830.

Green, J.T., \& Gay, F.H. (1956). Colles' fracture- residual disability. American Journal of Surgery, 91, 636-646.

Hansen, U., Zioupos, P., Simpson, R., Curey., J. D., \& Hynd, D. (2008). The effect of strain rate in the mechanical properties of human cortical bone. Journal of Biomechanical Engineering/Transactions of the ASME, 130, 011011-1 -011011-8. 
Holmes, A., \& Andrews, D.M. (2006). The effect of leg muscle activation state and localized muscle fatigue on tibial response during impact. Journal of Applied Biomechanics, 22, 275-284.

Houshian, S., Mehdi, B., \& Larsen, M. (2001). The epidemiology of elbow fractures in children: analysis of 355 fractures, with special reference to supracondylar humerous fractures. Journal of Orthopaedic Science, 6, 312-315.

Houshian, S., \& Andersen, H.M. (2000). Comparison of in-line and rollerskating injury. Scandinavian Journal of Medicine \& Science in Sports, 10, 47-50.

Hsiao, E.T., \& Robinovitch, S.N. (1998). Common protective movements govern unexpected falls from standing height. Journal of Biomechanics, 31(1), 1-9.

Hwang, I.K., Kim, K.J., Kaufman, K.R., Cooney, W.P., \& An, K.N. (2006). Biomechanical efficacy of wrist guards as a shock isolator. Journal of Biomechanical Engineering, 128, 229-234.

Jaffe, M.S., Dijekers, M.P., \& Zamentis, M. (1997). A population based survey of in-line skaters injuries and skating practices. Archives of Physical Medicine and Rehabilitation, 78, 1352-1357.

Jerosch, J., Heidjann, J., Thorwesten, L., \& Lepsien, U. (1998). Injury pattern and acceptance of passive and active injury prophylaxis for inline skating. Sports Medicine (Auckland, N.Z.), 6, 44-49.

Kim, K.J., Alian, A.M., Morris, W.S., \& Lee, Y.H. (2006). Shock attenuation of various protective devices for prevention of fall-related injuries to the forearm/hand complex. American Journal of Sports Medicine, 34, 637-643.

Kim, W., Voloshin, A.S., Johnson, S.H., \& Simkin, A. (1993). Measurement of the impulsive bone motion by skin mounted accelerometers. Journal of Biomechanical Engineering, 115, 47-52.

Lafortune, M.A., Henning, E., \& Valiant, G.A. (1995). Tibial shock measured with bone and skin mounted transducers. Journal of Biomechanics, 28, 989-993.

Lo, J., McCabe, G.N., DeGoede, K.M., Okuizumi, J.A., \& Aston-Miller, J.A. (2003). On reducing impact force in forward falls: results of a brief intervention in young males. Clinical Biomechanics (Bristol, Avon), 18, 730-736.

Milner, C.E., Ferber, R., Pollard, C.D., Hamill, J., \& Davis, I. (2006). Biomechanical factors associated with tibial stress fracture in female runners. Medicine and Science in Sports and Exercise, 38, 323-328.

Portney, L.G., \& Watkins, M.P. (2000). Foundation of Clinical Research Applications to Practice (2nd ed.). New Jersey: Prentice-Hall Inc.
Porta, D.J. (2005). Biomechanics of impact injuries. In J. Rich, D.E. Dean, \& R.H. Powers (Eds.), Forensic Medicine of the Lower extremity: Human Identification and Trauma Analysis of the Thigh, Leg, and Foot (pp. 279-310). Totowa, NJ: The Humana Press.

Public Health Agency of Canada. (2000). Injuries associated with in-line skating: CHIRPP database, summary data, all ages. Canadian Hospitals Injury Reporting Prevention Program. Retrieved on November 11 th 2007 from http:// www.phac-aspc.gc.ca/injury-bles/shirpp/injerep-rapbles/ inline_e.html.

Radin, E.L., Parker, H.G., Pugh, J.W., Steinberg, R.S., Paul, I.L., \& Rose, R.M. (1973). Response of the joints to impact loading-III. Journal of Biomechanics, 6, 51-57.

Ring, D., Jupiter, J.B., Sanders, R.W., Mast, J., \& Simpson, N.S. (1997). Translocation fractures of the olecranon. Journal of Orthopaedic Trauma, 11, 545-550.

Schieber, R.A., Branche-Dorsey, C.M., Ryan, G.W., Rutherford, J.W., Jr., et al. (1996). Risk factors for injuries from inline skating and the effectiveness of safety gear. The New England Journal of Medicine, 335, 1630-1636.

Staebler, M.P., Moore, D.C., Akelman, E., Weiss, A-P.W., Fadale, P.D., \& Crisco, J.J. (1999). The effect of wrist guards on bone strain in the distal forearm. American Journal of Sports Medicine, 27, 500-506.

Stewart, H.D., Innes, A.R., \& Burke, F.D. (1985). The hand complications of Colles' fractures. The Journal of Hand Surgery, 10-B, 103-106.

Tan, V., Seldes, R.M., \& Daluiski, A. (2001). In-Line skating injuries. Injury Clinic, 31, 691-699.

Troy, K.L., \& Grabiner, M.D. (2007). Off-axis loads cause failure of the distal radius at lower magnitudes than axial loads: A finite element analysis. Journal of Biomechanics, 40, 1670-1675.

Wake, H., Hashizume, H., Nishida, K., Inoue, H., \& Nagayama, N. (2004). Biomechanical analysis of the mechanism of elbow fracture-dislocation by compression force. Journal of Orthopaedic Science, 9, 44-50.

Wong, F.Y.H., \& Pho, R.W.H. (1984). Median nerve compression, with tendon ruptures, after Colles' fracture. The Journal of Hand Surgery, European Volume, 9, 139-141.

Wosk, J., \& Voloshin, A. (1981). Wave attenuation in skeletons of young and healthy persons. Journal of Biomechanics, 14, 261-267.

Zhang, S-N., Bates, B.T., \& Dufek, J.S. (2000). Contributions of lower extremity energy dissipation during landings. Medicine and Science in Sports, 4, 812-819. 